\title{
Metallicity and binarity in WC and WO stars
}

\author{
Laura Norci ${ }^{1}$, V. Francesco Polcaro ${ }^{2}$, and Roberto F. Viotti ${ }^{2}$ \\ ${ }^{1}$ Dunsink Observatory, Castleknock, Dublin 15, Ireland \\ ${ }^{2}$ Istituto di Astrofisica Spaziale e Fisica Cosmica, CNR, \\ Via Fosso del Cavaliere 100, I-00133 Roma, Italia
}

Median values of the FWHM and equivalent width $W_{\lambda}$ of the CIV $581 \mathrm{~nm}, \mathrm{OV}$ $559 \mathrm{~nm}$ and $465 \mathrm{~nm}$ emission lines were derived from an extended statistical analysis of WC and WO stars (Norci et al. 2002; see Table 1). We find that the C IV equivalent width shows an increasing trend towards earlier WC subtypes, reaching a maximum value of $95 \mathrm{~nm}( \pm 32 \%)$ for the Galactic WC4-6 stars, and decreases by about a factor of three in the hotter WO stars (Figure 1, left). The line appears about twice as strong in the LMC than in the Galactic WC4 stars, and the difference is particularly evident in the non-Galactic WO stars. Such an effect is absent in the Ov $559 \mathrm{~nm}$ line, which smoothly increases towards WCE and WO stars, with no significant difference between Galactic and non-Galactic WC4 and WO stars. Thus this C anomaly is not an ionization effect; it is, therefore, probably due to a cause that selectively operates on $C$ rather than on $O$. Different physical conditions in the winds of Galactic and non-Galactic stars, possibly related to the different initial metallicity $Z$, could be responsible for the anomaly.

The profile variation of the $465 \mathrm{~nm}$ emission, suggests a substantial contribution to the feature of He II $468 \mathrm{~nm}$ in the WO and WCE stars.

Composite-spectrum stars have line strengths that are systematically weaker, due to the presence of the additional continuum of the companion. Using the median values for single-spectrum stars, we have estimated the OB/WR continuum flux ratios in 25 WR binaries, some of which are listed in Table 2.

Table 1. Line median values (nm) in single-spectrum WC and WO stars.

\begin{tabular}{l|ccc|ccc}
\hline \hline subtype & \multicolumn{3}{|c|}{$\log W_{\lambda}$} & \multicolumn{3}{c}{ FWHM } \\
& $\lambda 581$ & $\lambda 559$ & $\lambda 465$ & $\lambda 581$ & $\lambda 559$ & $\lambda 465$ \\
\hline WO Galactic & $1.53 \pm .25$ & $1.27 \pm .20$ & $1.50 \pm .15$ & $13.5 \pm 1.3$ & $15.1 \pm 1.7$ & $9.7 \pm 1.1$ \\
WC4 & $1.99 \pm .21$ & $0.91 \pm .19$ & $1.81 \pm .17$ & $5.8 \pm .7$ & $5.8 \pm .6$ & $7.2 \pm .6$ \\
WC5 & $2.00 \pm .14$ & $0.73 \pm .19$ & $1.99 \pm .09$ & $4.5 \pm .9$ & $5.5 \pm .8$ & $5.3 \pm 1.0$ \\
WC6 & $1.96 \pm .14$ & $0.69 \pm .15$ & $1.99 \pm .11$ & $4.8 \pm 1.5$ & $4.7 \pm 1.3$ & $4.5 \pm 1.2$ \\
WC7 & $1.55 \pm .23$ & $0.53 \pm .07$ & $1.66 \pm .28$ & $4.6 \pm 1.5$ & $4.9 \pm .7$ & $4.6 \pm 1.3$ \\
WC8 & $1.46 \pm .14$ & $0.20 \pm .17$ & $1.63 \pm .13$ & $3.0 \pm .7$ & $3.0 \pm .7$ & $2.6 \pm .7$ \\
WC9 & $0.91 \pm .22$ & $0.25 \pm .27$ & $1.09 \pm .19$ & $3.2 \pm .3$ & - & $2.0 \pm .5$ \\
WC4 LMC & $2.27 \pm .11$ & $0.85 \pm .17$ & $2.19 \pm .09$ & $6.5 \pm 1.1$ & $5.7 \pm .7$ & $7.4 \pm .7$ \\
\hline \hline
\end{tabular}




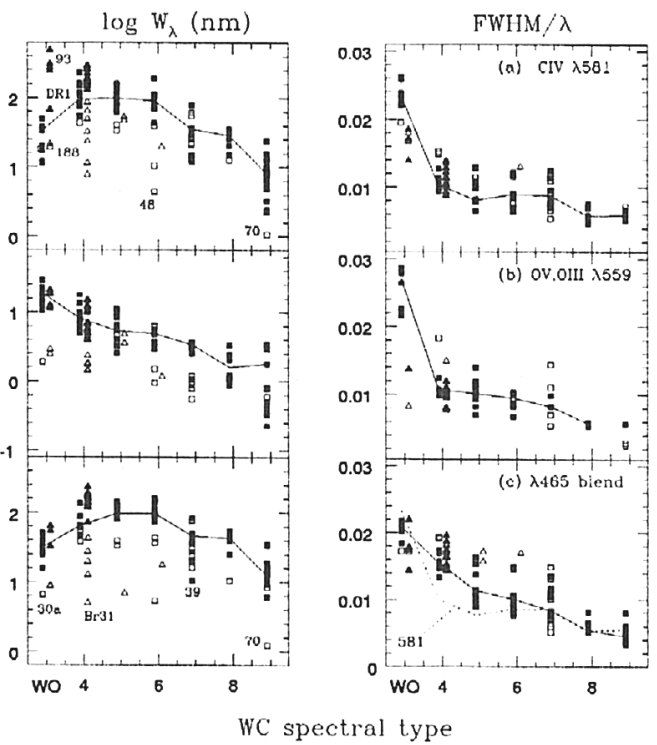

Figure 1. $\quad \log W_{\lambda}$ (left) and normalized FWHM (right) for Galactic (squares, solid lines) and non-Galactic (triangles) WO and WC stars, both in single (filled) and composite spectrum (open symbols) stars.

Table 2. $-\Delta \log W_{\lambda}$ and continuum flux ratio in composite-spectrum stars

\begin{tabular}{llllllll}
\hline \hline system & spectral type & $\lambda 581$ & $\lambda 569$ & $\lambda 559$ & $\lambda 465$ & ratio & error \\
\hline WR 30a & WO4+O5-5.5 & 0.27 & - & 0.99 & 0.68 & $\sim 3$ & $\mathrm{~d}$ \\
Sk188 & WO4+O7 & - & - & 0.78 & $0.8:$ & 6. & $\mathrm{c}$ \\
BAT99-34 & WC4+OB & 0.76 & - & 0.69 & 0.89 & 5. & $\mathrm{~b}$ \\
BAT99-38 & WC4+O8I: & 1.21 & - & $0.48:$ & 1.49 & $\sim 10$ & $\mathrm{~d}$ \\
BAT99-39 & WC4+O6V-III & 0.90 & - & 0.70 & 1.08 & 7. & $\mathrm{c}$ \\
BAT99-70 & WC4 & 0.45 & - & 0.60 & 0.63 & 3.0 & $\mathrm{~b}$ \\
WR 9 & WC5+O7 & 0.43 & 0.37 & 0.19 & 0.43 & 1.3 & $\mathrm{~b}$ \\
WR 30 & WC6+O6-8 & 0.37 & 0.36 & 0.71 & 0.36 & 1.3 & $\mathrm{~b}$ \\
WR 48 & WC6(+O9.5I/BoIab VB) & 1.31 & 1.15 & - & 1.26 & 16 & $\mathrm{~b}$ \\
WR 146 & WC6+O8 & 0.24 & - & - & 0.39 & 1.1 & $\mathrm{c}$ \\
WR 39 & WC7+OB? & 0.53 & 0.44 & - & 0.50 & 2.1 & $\mathrm{a}$ \\
WR 86 & WC7+B0III-I & 0.41 & 0.23 & 0.50 & 0.45 & 1.5 & $\mathrm{c}$ \\
WR 125 & WC7ed+O9III & 0.16 & 0.07 & 0.55 & 0.34 & 0.9 & $\mathrm{c}$ \\
WR 137 & WC7pd+O9 & 0.31 & 0.28 & 0.56 & 0.30 & 1.1 & $\mathrm{c}$ \\
WR 140 & WC7pd+O4-5 & 0.20 & 0.35 & 0.50 & 0.29 & 1.2 & $\mathrm{c}$ \\
WR 70 & WC9vd+BOI & 0.88 & 1.13 & - & 1.54 & 9. & $\mathrm{c}$ \\
\hline \hline
\end{tabular}

Notes: (1) error flags: a: <0.1, b: 0.1, c: 0.15, d: 0.2-0.3. Galactic WR spectral types from van der Hucht (2001); LMC WR spectral types from Breysacher et al. (1999).

\section{References}

Breysacher, J., Azzopardi, M., Testor, G. 1999, A\&AS 137, 117

van der Hucht, K.A. 2001, New Astron. Reviews 45, 135

Norci, L., Polcaro, V.F., Viotti, R.F., Rossi, C. 2002, RMxAA 38, 83 